\title{
Computerized Tomography Scanning and Magnetic Resonance Imaging Will Terminate the Era of the Autopsy - A Hypothesis
}

\author{
Daniel Benharroch ${ }^{\circledR}$, Shifra Shvarts ${ }^{2}$, Alan Jotkowitz ${ }^{3}$, Ilan Shelef ${ }^{4}$ \\ 1. Head of the Hematopathology Unit, Department of Pathology, Soroka University Medical Center and Faculty of Health Sciences, Ben Gurion University of \\ the Negev; \\ 2. The Moshe Privas Centre for Medical Education, Faculty of Health Sciences, Ben Gurion University of the Negev, Beer-Sheva, Israel; \\ 3. Department of Internal Medicine F., Soroka University Medical Center and Faculty of Health Sciences, Ben Gurion University of the Negev; \\ 4. Head of the Radiology Department, Soroka University Medical Center and Faculty of Health Sciences, Ben Gurion University of the Negev, Israel. \\ $\square$ Corresponding author: Prof. Daniel Benharroch, Institute of Pathology, Soroka University Medical Centre, P.O.Box 151, 1, Itshak Rager Blvd., \\ Beer-Sheva 84101, Israel. Tel: 97286400920. Fax: 97286232770. e-mail: benaroch@bgu.ac.il
}

(1) Ivyspring International Publisher. Reproduction is permitted for personal, noncommercial use, provided that the article is in whole, unmodified, and properly cited. See http://ivyspring.com/terms for terms and conditions.

Received: 2015.08.14; Accepted: 2015.11.01; Published: 2016.01.01

\begin{abstract}
Background: Reports on a marked reduction of the number of autopsies performed worldwide to less than $5 \%$ of hospital deaths remain without a satisfactory explanation. The premature disappearance of the autopsy might represent a medical tragedy of a major order. One of the causes for the decrease in autopsies is poorly documented: we suspect that the attending physician might show some reluctance when requesting a consent for an autopsy from the bereaved family. Moreover, this officer might consider that the post mortem will add little information to that already obtained from the computerized tomography scanner or the magnetic resonance imaging.

Methods: In order to confirm our hypothesis, we carried out a review of 300 articles indexed as "radiologic-histologic correlation", 118 of which were selected for a significant correlation. From the abstracts, we retrieved the type of the article, the degree of correlation as assessed by the authors and the form of imaging employed, and we computed them.

Results \& conclusions: The most striking correlation was observed in the small prospective series. An additional search for the "radiologic-autopsy correlation" supported a marked reduction in the number of post-mortems, especially those related with prospective studies. Based on the present study, we cannot determine precisely the role of the house officer in this tragedy. We may have demonstrated, however, that the modern radiologic methods have not yet reached a high enough performance quality to achieve the status of a candidate substitute for the autopsy.
\end{abstract}

Key words: Modern imaging methods; autopsy; history; biopsy; human factor; abstracts

\section{Introduction}

The deterioration of the autopsy status in Israel has been obvious for many years. The only adult post-mortems performed in this country, with some exceptions are forensic. The clinical autopsies still performed are those related to perinatal deaths. We instigated this study in order to learn from other countries, hoping their situation was less catastrophic, as they demonstrate some planning to prevent the "death of the autopsy".

\section{A brief history of the autopsy}

Ever since the hepatoscopy used in Mesopotamia to predict epidemics and other catastrophes by observing the liver of sacrificed animals, notions of anatomy were progressively introduced. During the following centuries, human anatomy made its first steps, notably in Greece and in Alexandria. This endeavour was limited by religion and by social objec- 
tion. Thus, it has been suggested that even Galen (130-215) elaborated most of his knowledge by dissecting higher apes [1].

One will have to await until the Renaissance (14 to $16^{\text {th }}$ centuries) to witness the renewal of human anatomy, culminating with Vesalius, the father of modern anatomy (1514-1562). Vesalius instituted human cadaver dissections against all the odds, mainly against religious banning. This period marked the ultimate enlightenment of anatomy $[1,2]$.

Following an era in which the European physicians developed the bases of physiology (Guido Guidi, 1509-1569 and Michel Servet, 1511-1553), the concept of physio-pathology was born to finally replace the teaching of Galen [1].

The marked progress in anatomy allowed the discovery of numerous anatomical variations that were for the first time interpreted as pathological modifications. Progressively, the anatomists made a habit of comparing, when possible, the symptoms and signs that preceded death with the gross changes observed after dying. From the global concept of the malady, established by Hyppocrate and later by Galen, the Renaissance anatomists developed in a somewhat nebulous momentum, the idea of the localization of a disease lesion to a specific organ $[1,3]$.

The thesis of the anatomy-pathology was sanctioned at the end of the $18^{\text {th }}$ century only. Autopsy meant originally: "seeing for one self". This period also marked the birth of "hospital medicine". The autopsy was blossoming, being the most effective of teachings. Bichat (1771-1802) stands out in his short existence, for having combined excellence as a clinician, a physiologist, a pathologist and as the first histologist [1].

The role of the autopsy in quality control [4] was later emphasized in the United States and elsewhere, when medical centres were rated according to the number of autopsies they performed [5]. However, in the late 1970s and the 1980s, a marked decrease in the number of post-mortems was noted. After a short revival, a persistent deterioration in the status of the autopsy lapsed to less than $5 \%$ of the hospital deaths [6].

\section{Factors suggested as a cause of the deteriora- tion of the status of the autopsy [7]}

Religion has been for long periods at the top of the list, ever since the Bull by Pope Bonifacius VIII (1299) and his ban of the human dissection [8].

Judaism is opposed to any interference with the dead. However, Rabbi Landau (18 ${ }^{\text {th }}$ century) ruled that if the dissection of a cadaver can contribute directly to the healthcare of a given patient, it should be authorized. In 1980, the Israel Knesset voted a law which endorses the autopsy, providing the written consent of three physicians and of a first degree relative of the patient. But, five hours should elapse between the death notification to the family and the start of the autopsy, to allow for a possible retraction. Practically, this law has made the rejection of the request by families much easier [8].

Islamic institutions stand basically against the autopsy. However, more recently, several Islamic organizations have initialized the re-examination of the official position on this issue. But fetuses and newborn babies dying around the time of birth are autopsied, as the health of future children in the family is at stake [8].

Social objection to the autopsy may vary widely in different communities in the same country. It relies often on maintaining the integrity of the individual and on his wishes and attitudes, as expressed before death [9].

Economical considerations regarding the autopsy are examined by countries, but also at the level of the individual hospital. It has been evaluated that the cost of an autopsy, including all its stages and which lasts at least 12 hours, is high. A ruling, by the authority, may justify the expense [10].

The fear of medico-legal consequences to the physicians has been evoked and so has the reluctance of the pathologists to perform autopsies [11].

Other factors contributing to the decrease in the number of autopsies are the substitution of paternalism for patients' autonomy as the dominant theme in the doctor-patient relationship. Thus physicians have much less latitude to unilaterally direct care and advocate for post-mortems, and patients are less likely to follow physicians recommendations blindly. This already tenuous relationship has been complicated by the medico-legal implications of modern medicine.

Before the event of modern imaging, several studies established that the clinical autopsies may disclose as many as $20 \%$ of diagnoses of major significance for the patients' morbidity and mortality, of which the attending physicians were unaware $[12,13]$.

\section{Basic modern imaging technologies}

The computerized tomography is a diagnostic imaging modality which uses $x$-rays to evaluate the projection of a target from all directions. The result, acquired as parallel axial slices, describes a 3-dimensional figure of the anatomy. Since its introduction in the 1970s, advances have occurred: helical scanning and multidetector row $\mathrm{CT}$, with as a consequence, improvement in the speed of imaging by several orders, and in the spacial resolution. The technique is more performing and represents the basis of medical imaging [14]. 
The magnetic resonance imaging originating in the late 1970s, has transformed medicine by contributing a non-invasive evaluation of tissue structure and function. However the MRI is a "difficult" and expensive technique. The cost is related to the duration of the examination. For thoracic MRI, the patient has to hold his breath, however dyspneic he may be.

The image data are not received directly, but through a $k$ space, related to the image space by Fourier transformation. MRI acquisition may take long, especially those with high resolution or wide coverage. If additional data can be led to stabilize the reconstruction of the image, we may get an acceptable image quality. This assumption is the basis of the partial Fourier imaging and parallel imaging, which are presently accessible, as well as of the compressed sensing reconstruction, which may in the future shorten the procedure and make it cheaper [15].

To our knowledge, the contribution of the computerized tomography (CT) and the magnetic resonance imaging (MRI) to the discordance mentioned above has not been evaluated thoroughly.

The reliability of the radiologist who uses the two modern imaging modalities in diagnosing a disease in hospitalized patients seems critical to the issue at hand. If, indeed, these radiologic modalities are highly dependable, then perhaps they might replace the autopsy in establishing a final and accurate diagnosis. In our opinion, the notion that this is indeed the case seems to have prevailed for several years already.

\section{Hypothesis: The modern imaging modalities are often considered a substitute for the au- topsy}

The 21rst century, in a world a priori more secular and richer should witness more autopsies and less antagonism to their application. Nevertheless, the distribution of wealth is highly unequal and as a consequence, religious activities are thriving. It is suggested that the reluctance of physicians in requesting an autopsy is not due only to timidity in the face of the bereaved family. It seems that most of them are confident that with the support of modern imaging, they possess all the answers they need on their patients' condition. Since the imaging studies are carried out on patients very much alive, there might be a discrepancy in the findings, when compared with the post-mortem diagnoses. However, that should be barred with the stipulation that they were carried out within a short range of time and that an autopsy was indeed performed.

At this point, a brief mention of the virtual autopsy is required. By this term, it is meant that modern imaging, the CT scan and the MRI are performed on a cadaver, to prevent the invasive procedure of the post-mortem [16]. Virtual autopsy is still in its infancy. In addition, the cost of the equipment, the space needed for their installation in the already crowded Pathology Institute, create a huge problem. On the other hand, using the scanners and MRI of the Imaging Institute for cadaveric imaging is not acceptable by most authorities.

To summarize, our hypothesis is that, due to religious, social and economic reasons, but also due to the attitude of the house officer, in many cases an autopsy is no longer being requested, perhaps as all the relevant diagnoses have been made by modern imaging methods.

\section{Validation of the thesis, using confirmation of the imaging by the biopsy}

Since the virtual autopsy has not been widely adopted, at least at this stage, one possible means of validating our thesis would be to compare the diagnoses of modern imaging, with those of the autopsy. Unfortunately, as less and less post-mortems are being performed and as time has elapsed since the imaging, this is not practical.

We may be left, therefore, with the closest alternative: we may have to compare, then, the imaging diagnosis with the report of the biopsy, when both are performed within a minimum lapse of time.

\section{Methods}

Three hundred articles were selected from PubMed for the definition: "radiologic-histologic correlation". The abstracts were analyzed and those not considered relevant enough for the correlation issue were discarded. The remainder were classified into the following categories: prospective series, retrospective series, reviews and case reports. The authors conclusions for each article was further divided into: good correlation and no correlation, if poor or nil. Last, the paper was tagged by the predominant imaging modality used.

The categories were determined, irrespective of the tissues or organs involved. The object of the imaging was, in part, non-neoplastic lesions, for example the MRI characteristics of cardiomyopathy. Alternatively, benign tumors were studied: small vessels hepatic haemangiomas or basal cell adenoma of the parotid gland. However, the vast majority of the diseases described were malignant neoplasms: lung and breast cancers, hepatocellular and renal cell carcinomas among others.

Comparison was carried out between the categories and between the subcategories and a $\chi^{2}$ test was performed.

In order to assess the rate of autopsies and the correlation which may be obtained with the radiologic 
diagnoses, we found 60 additional articles, selected for the index: "radiologic- autopsy correlation", between 2006 and 2015, and analyzed them as described for the first selection.

As no patient were involved directly with the study, but instead we collected the information from PubMed, we did not provide for a consent by the Ethics Institutional Committee.

\section{Results}

Of the first selection 300 articles were reviewed, only 118 were found adequate for our study (Figure 1). Eight subcategories were established (Table 1), two for each type of article - one demonstrated a good radiologic-histologic correlation; the other did not.

The category which showed the highest correlation was that of the prospective studies, it was also the smallest (18 articles), with a majority of very recent series (end of 2014 - beginning of 2015).

One of the prospective series examined 47 patients from France, with locally advanced pancreatic adenocarcinoma who were submitted to neoadjuvant chemo- and radiotherapy. The authors state that even partial reduction of the tumor-to-vessels contact, as shown by CT, was sufficient for the success of surgery [17].

Another prospective series of 40 patients with rectal cancer and complete mesorectal excision, showed $82.5 \%$ agreement between $\mathrm{T}$ stage by MRI and histology. In addition they found a 91\% correlation between imaging and individual lymph node histology [18]. These two articles are examples of papers on prospective series with a good radiological-histological correlation.

Table 1 shows a large group of retrospective series. Although the majority of these papers presented with a good radiologic-histologic correlation, the correlation was less significant than that for the prospective group $(\mathrm{p}=0.002)$.

The categories of Reviews and Case reports showed also an inferior correlation, when compared with the prospective series $(\mathrm{p}=0.0019$ and $\mathrm{p}=0.0023$, respectively). Seventeen of 18 (94.4\%) of the prospective series showed a good correlation. Twenty seven of 43 retrospective series $(62.8 \%)$ only showed a good correlation. Merely 18/38 (47.4\%) of the reviews, and only $8 / 19(42.1 \%)$ of the case reports presented with a good correlation. From a different perspective, only $17 / 70$ papers highlighting a good radiologic-histologic correlation were of the prospective variety $(24.3 \%)$. It is of note, that prospective series represented only 18 of 118 articles (15.25\%).

Only 14 articles were finally chosen from the second selection. In spite of the definition, no autopsy was performed in several categories of articles, and mainly in the most important, the prospective series. On the other hands, all the case reports were provided for by an autopsy.

\section{Discussion}

The objective of the present investigation was to attempt to validate our hypothesis, that, in the last several years, modern imaging (CT and MRI) is in the process of supplanting the autopsy, with regard to its role in determining clinical diagnoses. In other words, the clinicians, by concurring with the diagnoses of the CT scan and/or the MRI, may in fact decide that the option of the autopsy is unnecessary or even superfluous. An even worse development may be taking place: the house physician, fearing a medico-legal procedure if he was found to be mistaken in his diagnosis and treatment, rejoins the position of the radiologist. The result is that none of these officers did make the effort of obtaining the family consent, and even worse, they all finally lose interest in the procedure.

Table 1. Distribution of the articles selected by the: "radiologic-histologic correlation".

\begin{tabular}{lllllll}
\hline & $\mathrm{n}$ & $\mathrm{CT}$ & MRI both & else & $\mathrm{p}$ value \\
\hline Good correlation prospective series & 17 & 7 & 4 & 3 & 0 & \\
No correlation prospective series & 1 & 0 & 1 & 0 & 0 & \\
Good correlation retrospective & 27 & 8 & 14 & 2 & 3 & \\
series & & & & & & \\
No correlation retrospective series & 16 & 2 & 9 & 4 & 1 & 0.002 \\
Good correlation reviews & 18 & 6 & 6 & 5 & 1 & \\
No correlation reviews & 20 & 5 & 5 & 8 & 0 & 0.0019 \\
Good correlation case reports & 8 & 2 & 5 & 1 & 0 & \\
No correlation case reports & 11 & 1 & 9 & 1 & 0 & 0.0023 \\
TOTAL & 118 & & & & & \\
\hline
\end{tabular}

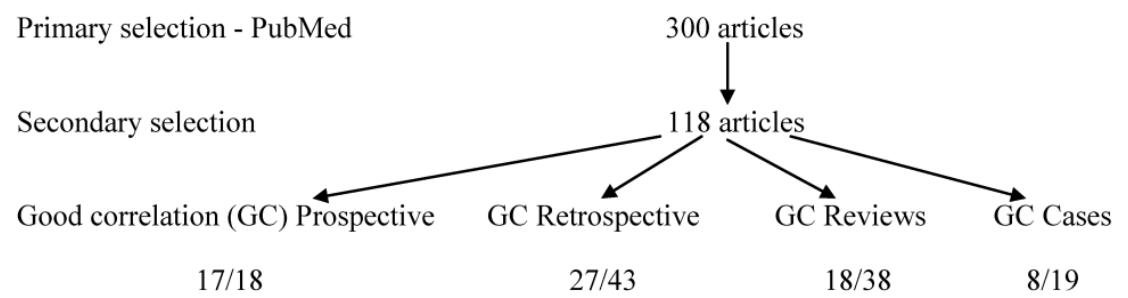

Figure 1. Study Selection flowchart - "radiologic-histologic correlations". 
This evolution, in which the autopsy standing is that of a "blind spot", if validated, may represent the last straw in a chain of events which may lead to the end of the era of the post-mortem. Since other causes are also evoked to the same effect, it may be very difficult to single out the role of the imaging techniques. Independently, the diminishing number of autopsies in many countries is a well established observation $[11,19,20]$. A large scale investigation with medical, sociological and epidemiological repercussions may be needed to determine the role of each factor in this issue.

We chose however to carry out a more restricted research. First, as we could not find more than a few papers correlating between modern imaging and the results of autopsies, and as we have excluded the virtual autopsy which is not yet mature for routine implementation, we decided to compare these radiologic examinations with the outcome of the biopsies. We were careful to limit the correlation to a reasonable time frame after the imaging.

Based on the definition: "radiologic-histologic correlation", we picked 300 consecutive articles. Of these, we selected 118 papers which were more directly concerned with the sought after correlation. Restricting ourselves intentionally to the abstracts of the articles, we retrieved three parameters, the category of the study, the authors' evaluation on the radiologic-histologic correlation and the radiological modality employed.

We found that the prospective series represented $15.25 \%$ of the 118 articles analyzed. However, $24.4 \%$ of these articles showed a good radiologic-histologic correlation. In fact, they stood for $24.3 \%$ of all the studies with a good correlation. Nevertheless, the prospective series with a good correlation represented $17 / 18(94.4 \%)$ of all the prospective articles.

Although retrospective studies with a good histological correlation represented $62.8 \%$ of the total retrospective articles, they showed a significantly lower correlation when compared with the prospective series. The proportion of good correlation review articles reached $47.4 \%$. They also represented a significantly lower correlation when compared with the prospective studies. Case reports showing a good histological correlation stand for $42.1 \%$ of the total reports. However, their radiologic-histologic correlation was poorer as compared with the prospective studies.

It appears that the radiologic-histologic correlation is the strongest in prospective series. This seems to point to this group as a leading category, the example to learn from if these radiological techniques are ever to be evaluated as pre-eminent. So far this does not seem to be the case since they represent less than a quarter of the studies with a good correlation.

Our second selection: "radiologic-autopsy correlation" probably supports our view that only few post-mortems had been performed in the period 2006-2015, showing poor correlation with the radiological findings. No autopsy was performed with the prospective studies. They were performed mainly with case reports.

Even though biopsies are only a surrogate to the autopsy, they helped disclose correlations with CT and MRI in several cases. Further limitations are made: our study contributed only modestly to the question of the pre-eminence of modern imaging among the causes of the deterioration of the status of the autopsy. Moreover, by comparing radiologic to histological diagnoses, we had to renounce an evaluation based on the sensitivity and the specificity of the techniques. In so doing, we have also implied that the anatomic pathological diagnosis is ultimately the most accurate; and this is not necessarily the case [21].

In conclusion, although we could not establish at this stage that modern imaging has supplanted the autopsy, it is obvious that in their present state, the modern radiologic modalities, as reflected by our study, did not show the qualities needed for them to be accepted as a candidate substitute for the autopsy. The role of the attending physician in the deterioration of the status of the autopsy is not conclusive either from our study. However, as this officer is found at the critical junction where the post mortem consent is acquired or lost, he should play an important role in this occurrence. More intricate investigations are needed.

Is it possible that virtual autopsy may eventually replace the post mortem? We may never know, as an evolution of this mixed radiologic-pathologic technology will necessitate a learning curve, meaning, at least at the start, clinical autopsies conducted in parallel and after each virtual autopsy. And this brings us back to the original query.

\section{Evidence added in proof}

We have identified an additional index which may shed a different light on the issue at hand: " the clinical-autopsy discrepancy". This definition underlines the clinical diagnoses, of which the radiologic studies are only one part.

A marked discordance was found in one article, describing several acute conditions, myocardial infarction, pulmonary thromboembolism, aortic aneurysm rupture etc... Major discrepancies were found in $23.5 \%$ of the autopsies [19], meaning that, at least in some medical centres, the persistence of a similar rate of discordance as found in the era preceding the use of modern imaging could still be identified. 
A similar study [20 ] showed in 2010 a discrepancy between ante-mortem and post-mortem diagnoses of $23.1 \%$. However only 591 autopsies out of more than 6,300 disclosed enough data to be included in the paper, showing that even in medical centres with high autopsy rates, this procedure is considered with lack of reliability.

A multicentre study from Berlin, in 2012 [22], which noted a deterioration to $3.5 \%$ in the rate of autopsies revealed a decline of $10.7 \%$ in the discordance, from $43.4 \%$ in 1988 to $27.1 \%$ in 2008 . A further article showed differential discordance rates of $27.8 \%$ in medical intensive care; of $32.7 \%$ for the surgical service and $31.3 \%$ for nursing home [23].

\section{Acknowledgement}

The study was carried out during D. Benharroch's sabbatical and no additional specific expense was necessary.

\section{Competing Interests}

The authors have declared that no competing interest exists.

\section{References}

1. Dachez R. Histoire de la medecine. De l' Antiquite a nos jours. Tallandier Editeur. 2012, Paris: p. 25; pp 99; pp 209; pp 357; p. 374; pp 477; pp 526

2. Gulczynski J, Izycka-Swieczewska E, Grzybiac M. Short history of the autopsy. Part I. From prehistory to the middle of the 16th century. Pol J Pathol 2009;2:109-114. - Part II. From the second half of the 16th century to contemporary times. Pol J Pathol 2010;3:169-72.

3. Dachez R. Histoire de la medecine. De l'Antiquite a nos jours. La methode anatomo-clinique. Tallandier Editeur. 2012, Paris: pp533.

4. Petros K, Wittekind C. Autopsy - a procedure of medical history? Med Klin Intensivmed Notfmed 2014; 109:115-20.

5. King LS, Meehan MC. A history of the autopsy. A review. Am J Pathol 1973;73:514-44.

6. Burton JL. A bite into the history of the autopsy: From ancient roots to modern decay. Forensic Sci Med Pathol 2005;1:277-84.

7. Zhu MH, Yu DH. Fluctuations in the rate of autopsy in China. Chin Med J (Engl) 2011;124:3403-7.

8. Henry J, Nicolas N. Dead in the water - - are we killing the hospital autopsy by poor consent practices? J R Soc Med 2012;105:288-95

9. Eka I, Rowan C, Osborn M. Mind the gap: are NHS trusts falling short of recommended standards for consent to autopsy? J Clin Pathol 2014;67:10-3.

10. Royal College of Pathologists of Australasia Autopsy Working Party. The decline of the hospital autopsy: a safety and quality issue for healthcare in Autralia. Med J Aust 2004;180:281-5.

11. Sblano S, Arpaio A, Zotti F, et al. Discrepancies between clinical and autoptic diagnoses in Italy: evaluation of 879 consecutive cases at the "Policlinico of Bari" teaching hospital in the period 1999-2009. Ann Ist Super Sanita 2014;50:44-8.

12. Battle RM, Pathak D, Humble GC, et al. Factors influencing discrepancies between premortem and postmortem diagnoses. JAMA 1987;258:339-44.

13. Veress B, Alafuzoff I. A retrospective analysis of clinical diagnoses and autopsy findings in 3,042 cases during two different time periods. Hum Pathol 1994;25:140-5.

14. Pelc NJ. Recent and future directions in CT imaging. Ann Biomed Eng. 2014;42: 260-8.

15. Hollingsworth KG. Reducing acquisition time in clinical MRI by data undersampling and compressed sensing reconstruction. Phys Med Biol. 2015;60:R297-R322.

16. Westphal SE, Apitzsch J, Penzhofer T, et al. Virtual CT autopsy in clinical pathology: feasibility in clinical autopsies. Virchows Arch 2012;461:211-9.

17. Cassinotto C, Mouries A, Lafourcade JP, et al. Locally advanced pancreatic adenocarcinoma: reassessment of response with $\mathrm{CT}$ after neoadjuvant chemotherapy and radiation therapy. Radiology. 2014;273:108-116.

18. Park JS, Jang YJ, Choi GS, et al. Accuracy of preoperative MRI in predicting pathology stage in rectal cancers: node-for-node matched histopathology validation of MRI features. Dis Colon Rectum. 2014;57:32-8.
19. Kuijpers CC, Fronczek J, van de Goot FR, et al. The value of autopsies in the era of high-tech medicine: discrepant findings persist. J Clin Pathol 2014;67:512-9.

20. Moorchung N, Singh V, Mishra A, et al. Is necropsy obsolete - an audit of the clinical autopsy over six decades: a study from Indian subcontinent. Indian J Pathol Microbiol 2013;56:372-7.

21. Saracci R. Is necropsy a valid monitor of clinical diagnostic performance? BMJ 1991;301:898-900.

22. Wittschieber D, Klauschen F, Kimmritz AC, et al. Who is at risk for diagnostic discrepancies? Comparison of pre- and postmortal diagnoses in 1800 patients of 3 medical decades in East and West Berlin. PLoS One 2012;7:e37460.

23. Scordi-Bello IA, Kalb TH, Lento PA. Clinical setting and extent of premortem evaluation do not predict autopsy discrepancy rates. Mod Pathol 2010;23:1225-30. 